\title{
Erratum: Observation of Sequential $\Upsilon$ Suppression in PbPb Collisions [Phys. Rev. Lett. 109, 222301 (2012)]
}

\author{
S. Chatrchyan et al. \\ CMS Collaboration
}

(Received 8 April 2018; published 11 May 2018)

DOI: 10.1103/PhysRevLett.120.199903

The global uncertainty in the double ratios (the size of which is represented by the size of the box placed at 1 , on the right vertical axis in Fig. 2 (left) has been increased by roughly a factor of $2(13 \%-24 \%)$. This global uncertainty represents the uncertainty on the $p p$ ratio, which is the common denominator for all the points shown in Fig. 2 (left). The change fixes using the absolute uncertainty on the $p p$ ratio $(0.13)$ as a relative uncertainty. The uncertainty box has been updated based on the revised uncertainty, as has the table in the Supplemental Material. The corrected figure is given below and the Supplemental Material has been updated (Ref. [18] of the Letter). The conclusion of the paper is not affected by this change.
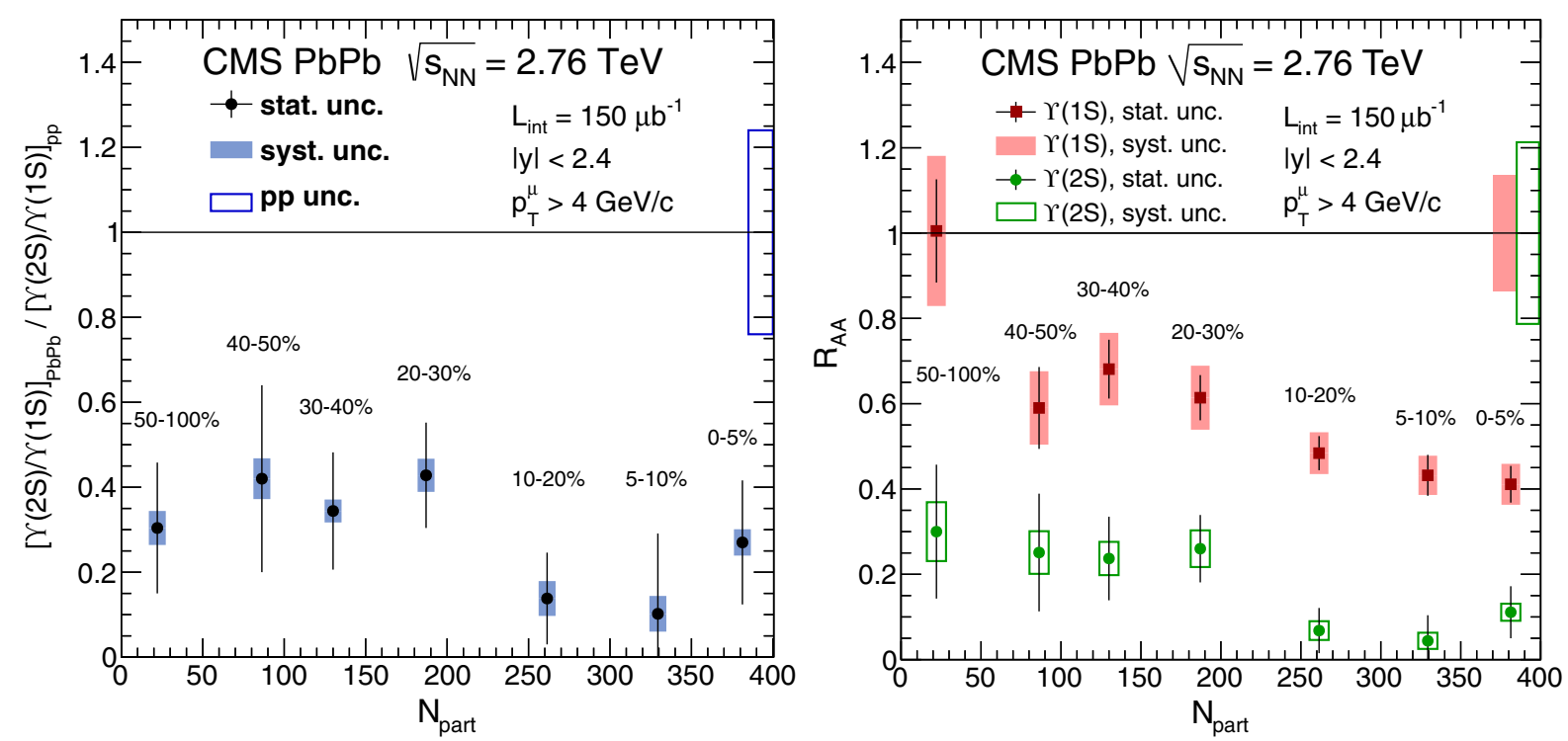

FIG. 2. Centrality dependence of the double ratio (left) and of the nuclear modification factors (right) for the $\Upsilon(1 S)$ and $\Upsilon(2 S)$ states. The relative uncertainties from $N_{\text {part }}$-independent quantities ( $p p$ yields and, for the $R_{\mathrm{AA}}$, also integrated luminosity) are represented by the boxes at unity, and are not included in the data points as these uncertainties do not affect the point-to-point trend. The event centrality bins used are indicated by percentage intervals. The results are available in tabulated form in the Supplemental Material.

Published by the American Physical Society under the terms of the Creative Commons Attribution 3.0 License. Further distribution of this work must maintain attribution to the author(s) and the published articles title, journal citation, and DOI. 